Original article

Received: 13 February 2017 / Accepted: 19 April 2017

\title{
THE NEW APPROACH OF USING IMAGE AND RANGE BASED METHODS FOR QUALITY CONTROL OF DIMENSION STONE
}

\author{
Volodymyr Levytskyi \\ Department of Mine Surveying, Zhytomyr State Technological University, \\ Zhytomyr, Ukraine
}

\begin{abstract}
The basis for the quality control of commodity dimension stone blocks for mining industry is the study of fracturing. The identification of fracturing in rock masses is one of the most important aspects in rock mass modelling. Traditional methods for determination properties of fracturing are difficult and hazardous. This paper describes a new approach of fracturing identification, based on image and range data, which realized by image processing and special software. In this article describes a method using new computer algorithms that allow for automated identification and calculation of fracturing parameters. Different digital filters for image processing and mathematical dependences are analyzed. The digital imaging technique has the potential for being used in real time applications. The purpose of this paper is the accurate and fast mapping of fracturing in some walls of the Bukinsky gabbro deposit.
\end{abstract}

Keywords: dimension stone, fracturing, photogrammetry, image processing, quality control, mine surveying

\section{Introduction}

Dimension stone is a collective name used for various natural stones that have been quarried and trimmed to specific sizes or shapes for structural and decorative purposes in buildings, interior and exterior applications. In Zhytomyr region more than 300 deposit of dimension stone such type of hard stone as granite, labradorite, gabbro, granodiorite, syenite and others. The main dimension stone product type are blocks, slabs, tile, countertops, paving stone, curbs, monuments.

The analysis of mining operations complex of mining companies demonstrates necessity of implementation for production of new technologies, which one would provide automation of operations, have augmented performance of mining operations and have enhanced trust to results of measurements and declared quality of raw by the producer. Automation it is recommended to carry out on the basis of use of 
remote-sensing methods of estimation fracturing of rock mass, new digital instruments, modern technological complexes of the equipment for dimension stone extraction and the applicable software.

For the mining industry, the large fracture databases can be used to optimize the many costly and energy-consuming rock breaking steps, including cutting, crushing and grinding. For the mining industry, the large fracture databases allow probabilistic engineering design to be carried out on a regular basis on projects where such a detailed analysis was not cost effective previously. Also, by imaging rock faces from a distance, both safety and access problems are eliminated (Kemeny et al., 2002).

Researches of modern equipment for surveying operations (Jing, 2003) has resolved to define effective variant which one provides not only necessary accuracy and efficiency of computer model creation, but also the problem solving, which linked to conducting of high-precision measurements. For surveying of dimension stone monoliths and blocks, identification of cracks in rock mass with following definition of their geometrical parameters, the most effective is using of the modern digital cameras and terrestrial laser scanning (Zawieska \& Markiewicz, 2015).

More recently, laser range (Feng \& Roshoff, 2004) and three-dimensional photogrammetry (Poropat, 2001, Haneberg, W.C. 2008) have been employed. Both techniques offer high accuracy and efficiency over manual measurements (Coggan, 2007, Delis, 2016). Advantages of laser and photogrammetric methods for quality control of dimension stone are several:

- the ability to collect large data sets yields a more realistic picture of the fracture orientations, new fracture sets are oftentimes discovered that would be missed by manual investigations.

- the ability to analyze large portions of rock masses, including inaccessible areas.

- the ability of zooming in and out of the rock face allows one to identify features, such as major shears or fractures, which are otherwise not apparent when working close to the rock face.

- new insights into the rock mass structure are gained by selecting fractures or fracture sets on a stereonet and seeing their location on the 3D model.

- these methods are faster than manual data collection, without taking into account the much larger number of data points.

- permanent documentation of the rock face condition for reporting and contractual-legal issues.

The main aim is developing of the numerical analysis technique of stone cracks system which is basis for recognition of photos or scans elements and optimization of main parameters, which influence on quality of rock mass optical control. The purpose of this paper is the accurate and fast fracturing mapping in some walls of the Bukinsky gabbro deposit.

\section{Related Works}

Traditional methods for obtaining fracturing data in the field include the scanline survey and cell mapping (Priest, 1993). In the scanline survey, fracture information is collected along a line at a rock face. Many computer approaches have been developed for estimating three-dimensional fracture orientations from twodimensional fracture trace information gathered from digital images of exposed rock faces (Kemeny \& Post, 2003). Kemeny has combined the hough transform and edge 
detection algorithms for joint tracking from digital images, proposing a simple classification using the detected traces angles (Kemeny \& Mofya, 2002). Lemu and Hadjigeorgiou have presented a digital face mapping methodology to construct the discontinuity trace maps from photographs of rock faces (Lemy \& Hadjigeorgiou, 2003).

Sobolevskyi has investigated relationship between the number of cracks in the rock mass and their spatial orientation for selection the direction of mining development (Sobolevskyi et al., 2016).

Rock masses were mapped and analyzed using linear mathematical transformations and isometric perspective methods to achieve meaningful threedimensional results in research by Turanboy and Ülker, 2010.

Photogrammetric systems and terrestrial laser scanners have high potential for measuring stone fracturing. It is caused by progress in field image processing and possibility of application of intellectual methods of an image processing (Wang \& Huang, 2010; Yamaguchi \& Hashimoto, 2009; Dare et al., 2002) which one resolve automatically to define with high accuracy of object characteristic dots coordinates.

The results obtained show that digital image analysis method has a very good accuracy in assessing the fine structures, and also it collects data in a much shorter time (Mohebbi et al., 2017).

A software for analyzing the geometric characteristics of fracturing in a rock mass was developed by Turanboy \& Ülker, 2010 and González-Aguilera et al., 2016.

\section{Materials}

\subsection{Theoretical part}

In fractured rock outcrop there should be selected an area of a square shape, the size of which depends on the nature, amount and density of fractures. The studied area is to be photographed and all fractures found within it numbered and described. All fractures are divided by genesis, width and character of development into several groups described by number of fractures and their length. Length of fractures should be calculated by group, taking the ratio (\%) of the total length of fractures to the area of counting, which gives us figures of specific fracture.

Development of fracture network numerical analysis methods is based on the identification of still images elements and optimizing key input parameters that affect quality of optical monitoring of natural stone array.

Usually images formed by different information systems are distorted by noise effects. It makes difficulties in image analysis and automated processing. Reducing of noise or complete its exclusion is achieved by filtration. Linear action, median and adaptive filters are the basis for choice of noise reducing method. After analyzing and comparing the work of the above mentioned methods of noise reduction median filtering was chosen as the best option for image processing.

As the linear rectifier filter the average filter which original value is mean value on the suburb of a mask of the filter is used. This filter is described by the following dependence (Gonzalez \& Woods, 2008):

$$
G_{i, j}=\sum_{s=\frac{(m-1)}{2}}^{(m-1) / 2} \sum_{t=\frac{(n-1)}{2}}^{(n-1) / 2} W_{s, t} \cdot E_{(i+s),(j+t)},
$$


where $G_{i j}$ - array element of image after filtering; $W_{s, t}$ - mask element of filter, which has a size $m \times n ; s \in[-m / 2, m / 2], t \in[-n / 2, n / 2]$ - coordinates respectively on an abscissa axis and ordinate; $E_{i j}$ - array element of the source image.

Analyze the work of the median filter based on mathematical correlations. The median filter provides nonlinear filtration of noises in the image and is scanning the mask covering an odd number of samples.

The median filter replaces the central mask element of the ordered median selection formed of all amplitudes of samples that are covered by the mask filter. When applying a median filter there is sequential processing of every point of the frame resulting in a sequence of estimates. Thus, a two-dimensional median filter box with central symmetry is used as a mask, with its center located at the current point of filtering. The median filter is described by following dependence:

$$
G_{i, j}=\operatorname{med}\left[E_{i+s, j+t} ;(s, t) \in W_{s, t}\right] .
$$

At the base of the adaptive filtering it is necessary the vinerivsky filter which is one of types of the linear filter for the adaptive local processing of the image. If value of root mean square deviation of intensity of pixels in this local area big, then the filter executes insignificant smoothing and, on the contrary, in case of a smaller deviation the field of smoothing increases. This approach is more effective, than normal linear filtering.

Mean value of brightness of the central pixel of a mask $W_{i, j}$ is calculated on a formula:

$$
\bar{w}=\frac{1}{m \cdot n} \cdot \sum_{i=1}^{m} \sum_{j=1}^{n} W_{i, j} .
$$

Dispersion of a mask:

$$
\delta^{2}=\frac{1}{m \cdot n} \cdot \sum_{i=1}^{m} \sum_{j=1}^{n}\left(W_{i, j}-\bar{w}\right)^{2} .
$$

In this formula for each new provision of a mask the appropriate values repeatedly are calculated. Deleting noise is estimated through an average quadratic deviation.

The purpose of binarization operation is radical reduction of amount of information on the image. In the course of a binarization the source 16-bit image turns in blackand-white which pixels have only two values -0 and 1 . The principal parameter of such conversion is the threshold - value which will be criterion of image point intensity check.

The adaptive binarization is used for image processing on which through nonuniformity of a background the normal binarization has bad results. Among methods of the adaptive binarization select the following: Niblack, Sauvola, Chistian, Bernsan (Gonzalez \&Woods, 2008).

The universal method or algorithm for the solution of all types of tasks of segmentation doesn't exist, each of methods has the advantages and shortcomings. Therefore, in operation the comparative analysis of methods of segmentation of the existing segmentation methods is made.

For comparing of methods of segmentation of digital images the appropriate masks which principle of operation is based on a difference of brightness of elements and a background of the image were simulated. Mathematical reasons for methods consist in calculation of the derivatives presented for digital images in the form of the discrete approaches of a gradient. As gradients operators (matrix masks) among 
whom select the following are used: Robert's Crossoperator, Prewitt method, Sobel operator and operator Laplacian of Gaussian (LoG).

One more method of segmentation is the method of Canny method (Canny, 1986), which essence consists in search of local sections with overfalls of brightness. Overfalls of brightness look for on each of coordinates axes a one-dimensional mask of Sobel or Laplasian of Gausian. For images with noise this method provides the best detection of boundaries in comparison with other methods, but requires significantly bigger time.

As the previous stage of segmentation is the adaptive binarization, in case of a choice of an optimal method of the digital image segmentation it is necessary to consider influence of parameters of the adaptive binarization considered methods for this purpose these image processing stages are considered in a complex.

Resulting a set of the segments received during the previous computation may contain insignificant fragments or not fully to reflect the provision of cracks. Therefore, from it segments are deleted, a certain threshold value is shorter. The choice of threshold value of length of a crack is carried out, proceeding from density of cracks layout and amount of not filtered sound effects. At this stage the user has an opportunity to set segment length, isn't considered.

\subsection{Methodology and experiments}

For the analysis of fracturing the developed by the author software "Crack Stone», which allows to analyze a qualitative camp of rock mass and it fracturing. The Borland $\mathrm{C}++$ programming language was used in the software. An example of the initial photos of rock mass of Bukinsky deposit of gabbro (Ukraine), which was fixed in software «Crack Stone», presented in Fig. 1.

The basic quantity indicators of an estimation fracturing is specific plane fracturing and specific linear fracturing which provide the fullest information about destruction of mass. For this reason, as a result of image processing of dimension stone mass, the area, quantity and length of cracks on an investigated site are defined.

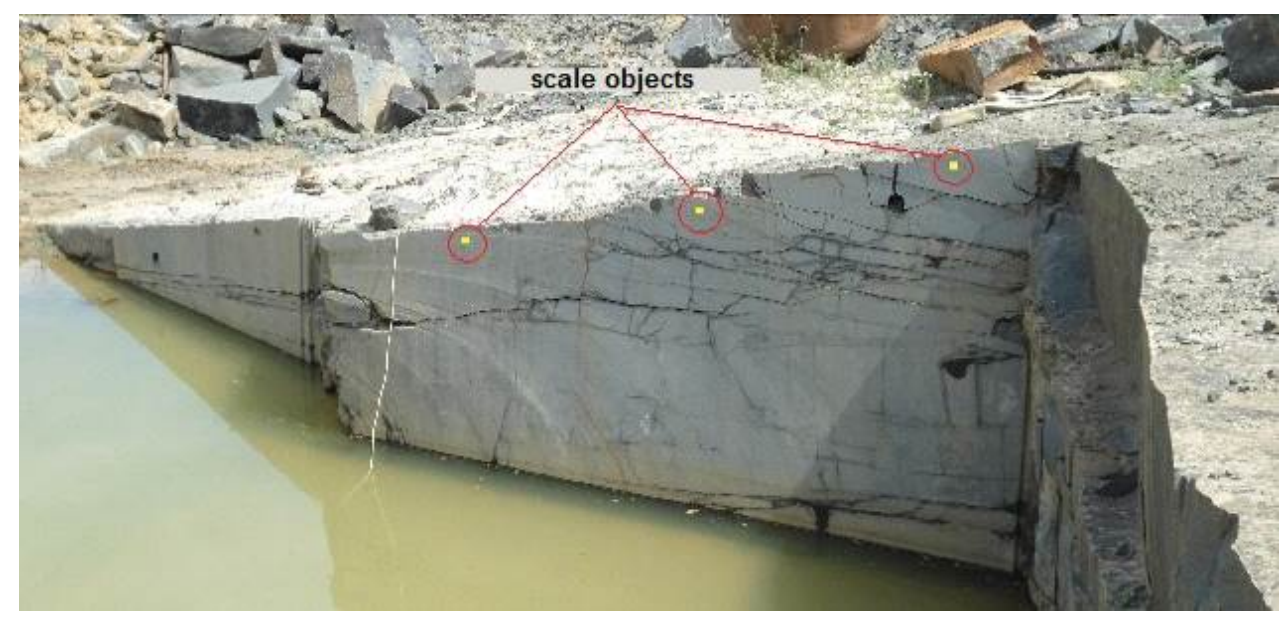

Fig. 1. The subject of surveying - cracks in rock mass of Bukinsky deposit of dimension stone 
Algorithm of program for image analysis and fractures recognition "Crack Stone" is described in Fig. 2. Let's look through the stages of program work according to its algorithm. Digital photo shown in Fig. 1 is selected and opened as background information.

Scaling is performed by the scale tool (ruler or leveling rod) or other object with a known size. Then we should input dimensions and scaling units and cut the zoom area of the photo we want to analyze. The image is processed by different filters and at every stage of processing it is possible to see the results filtering with the specified parameters, which allows us to choose the optimum settings for a particular shot.

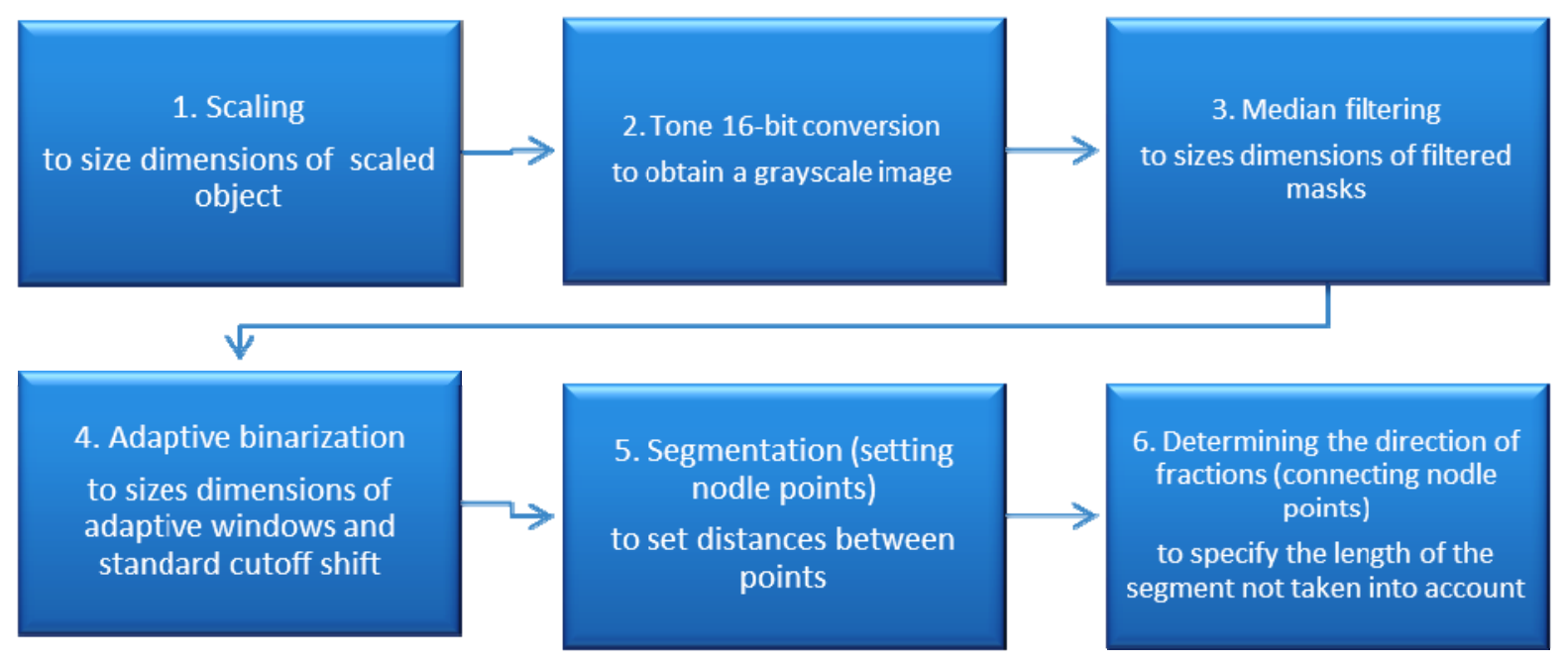

Fig. 2. The algorithm of the program «Crack Stone»

In Fig. 3 results of filtering are given by the types of filters considered above when superimposing noise on the digital image with cracks in an array of a natural stone.

Filtering noise on gray and black-and-white of each filter (Fig. 3) shows the degree of artifacts manifestation and distortions in black-and-white images that cannot be seen on gray images.
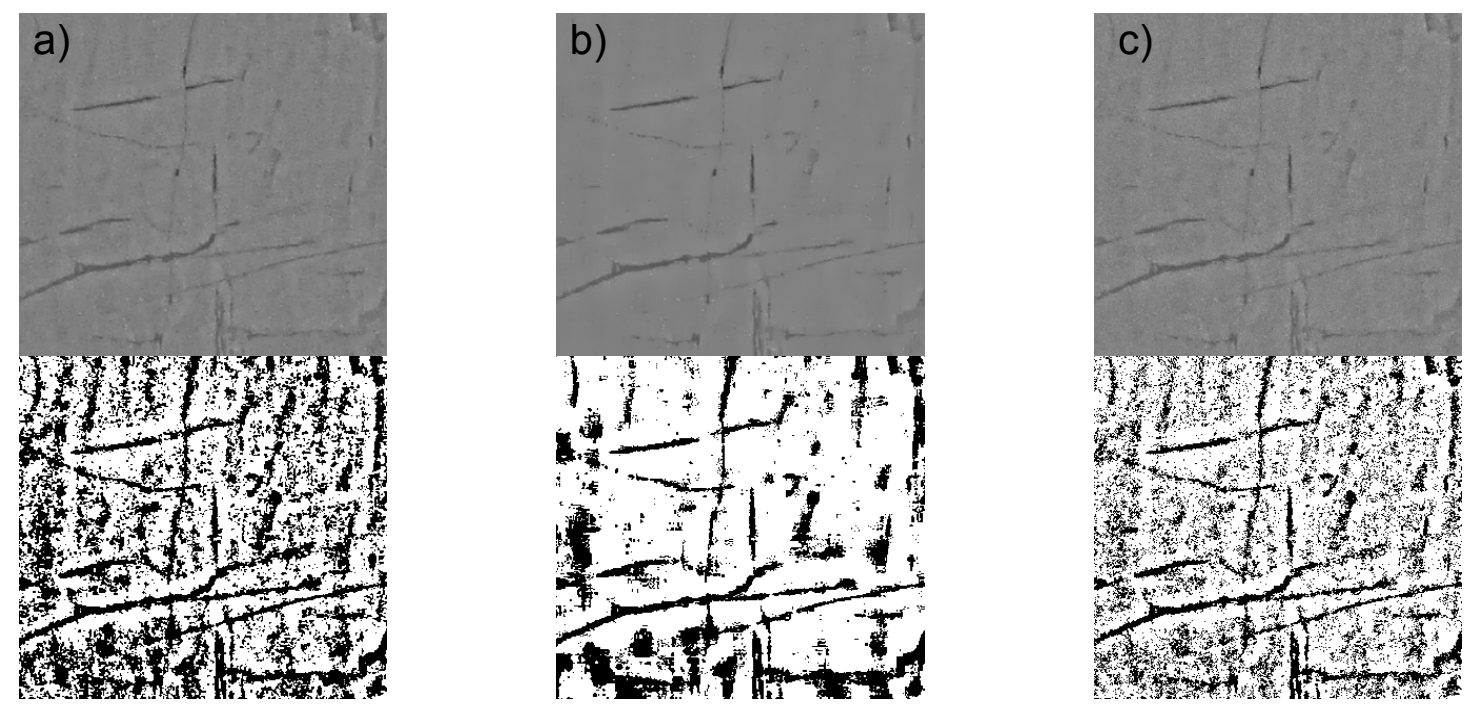

Fig. 3. Results of filtering noise on gray and black-and-white images of an array with cracks: a) the linear filter; b) median filter; c) the adaptive filter. 
The analysis of Fig. 4 shows that deleting noise by the median filter is characterized by bigger efficiency in comparison with other filters as leads to smaller distortion of the image subjects boundaries.

So, being guided by the above-stated analysis, in the application the median filter which destroys noise on the image is realized, reduces quantity of the small isolated fragments and accelerates further processing stages of the image. At this stage the user has an opportunity to set the sizes of the median filter mask (Fig. 4,a).
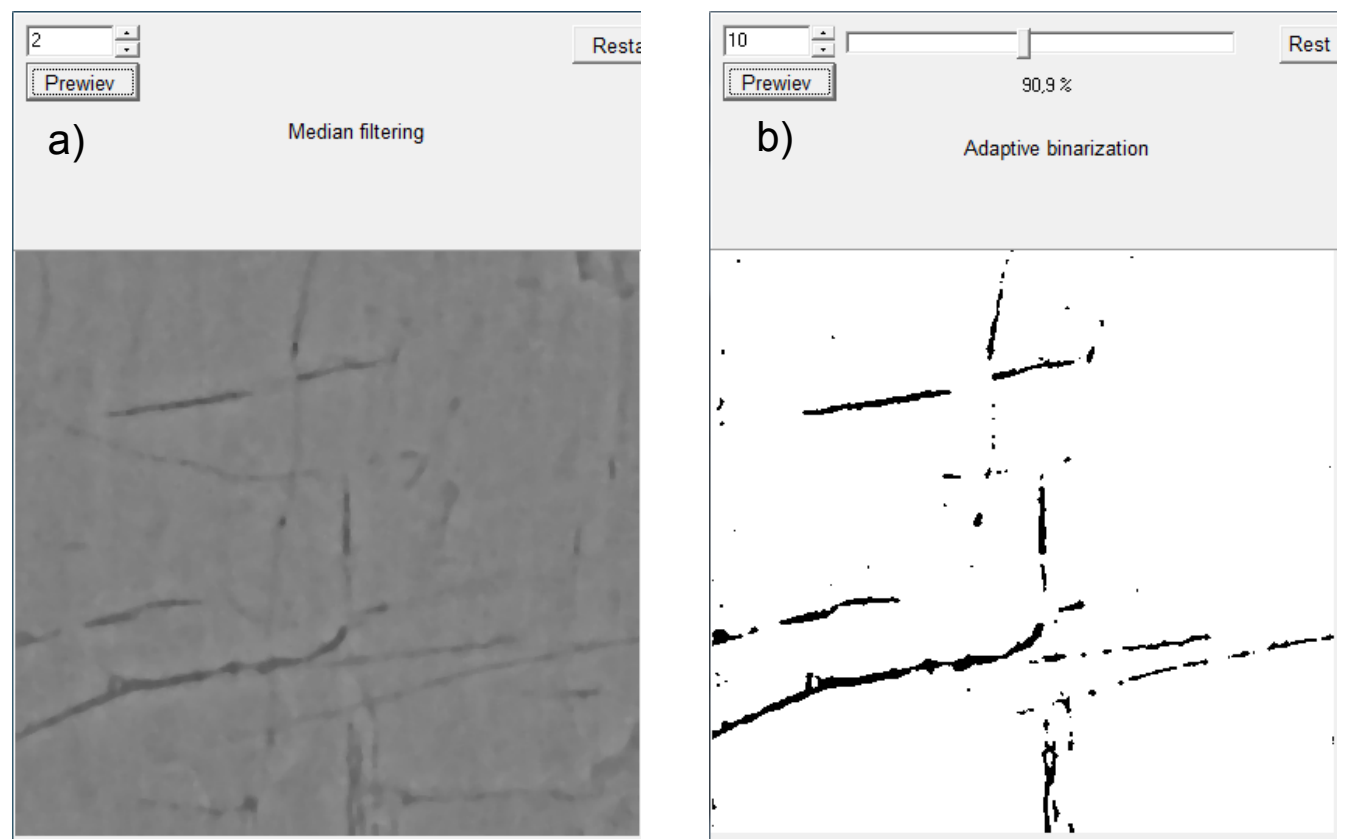

Fig. 4. Results of median filtering (a) and the adaptive binarization (b).

As the physical nature of cracks the same, it is possible to pick up such value of a threshold (background boundary) in case of which the specified conversion with a high level of reliability will allow to reveal boundaries of cracks on the source image. Results of operation of the adaptive binarization are shown in Fig. 5. In operation it is offered to calculate a threshold by Christian's method. At this stage the user has an opportunity to set the sizes of the adaptive window and offset of a standard threshold.

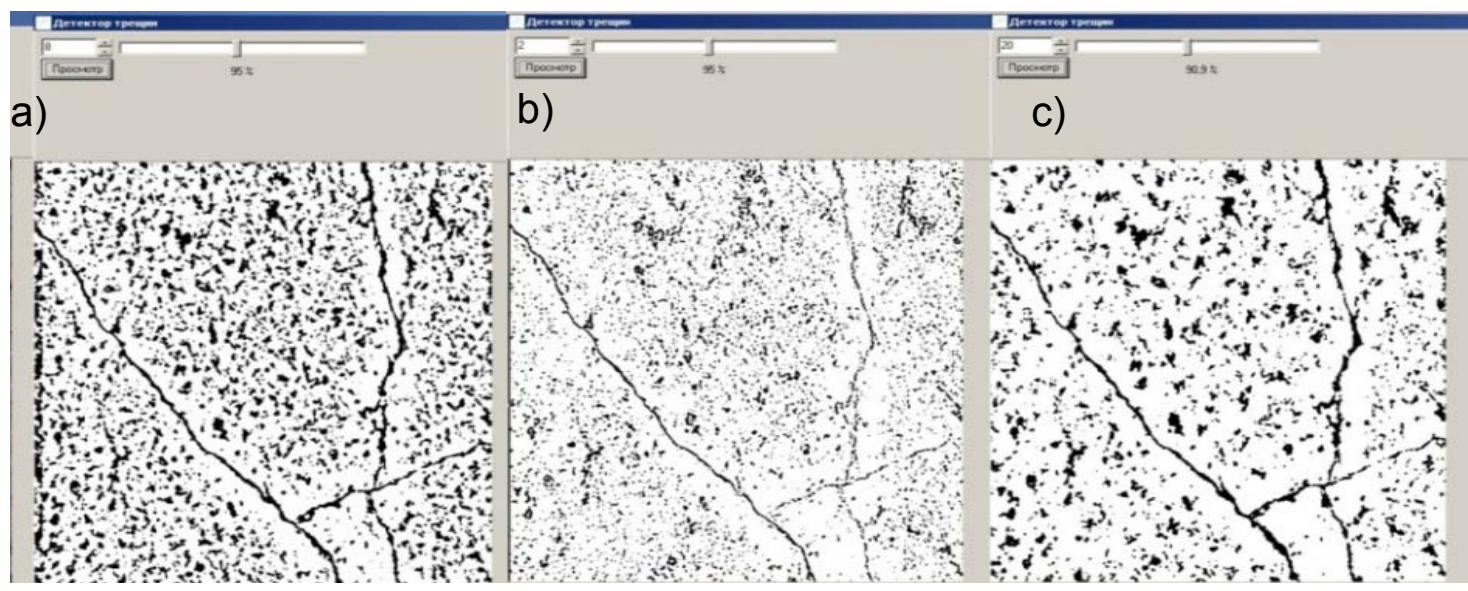

Fig. 5. The results of an adaptive binarization of digital photos of cracks for deeps:

a) $8 \mathrm{~mm}$; b) $2 \mathrm{~mm}$; c) $20 \mathrm{~mm}$. 
In case of a choice of the median filter parameters and the adaptive binarization it is recommended to reach the minimum thickness of cracks and at the same time their continuity. In this case the most exact results will be received.

The end result of the image analysis is also defined by quality of segmentation which is the following processing stage and consists in preliminary separation of an object circuits which is identified. Algorithms of images segmentation are based on one of two characteristics of a brightness signal - discontinuity or homogeneity. In the first case approach is based on partition of the image on the basis of a signal sharp changes, such as image brightness overfalls. Usually search of gaps is carried out by means of special masks. As methods of detection of overfalls derivatives and gradients from functions of brightness are used. The second category of methods is based on determination of the image homogeneity according to in advance selected criteria.

Results of the digital image segmentation by means of the considered algorithms are shown in Fig. 6.

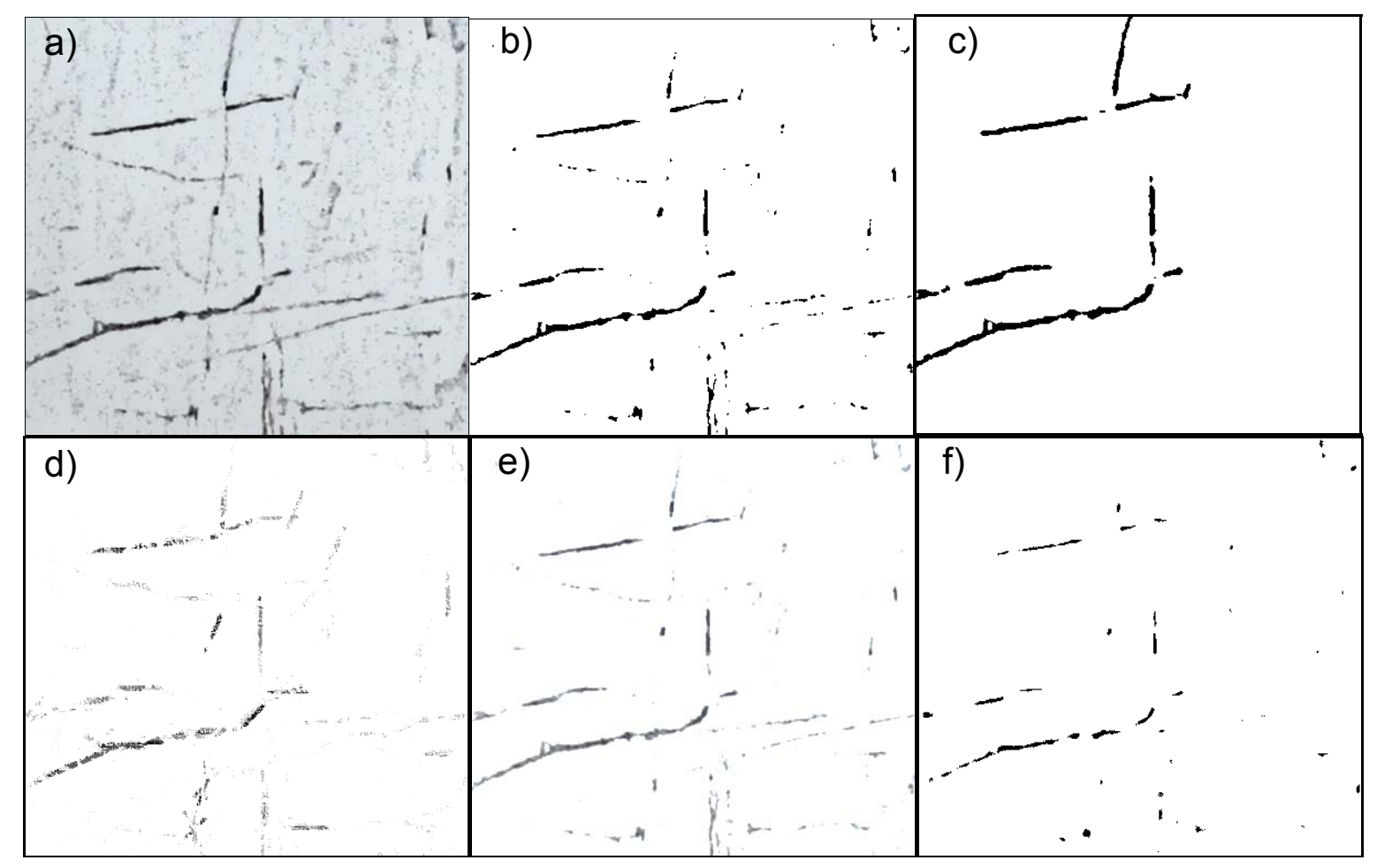

Fig. 6. Results of segmentation of the digital image:

a) source image; b) operator Laplasian of Gausian; c) Kenny's method;

d) Roberts's operator; e) Previtt's operator; e) Sobel's operator

In parallel with segmentation in the application arrangement of nodal points which allows to divide the image into component fragments is executed and to reveal, thus, the sections belonging to certain cracks. Nodal points are put not through a certain step, and in each pixel, and at the same time there is a check for existence of already existing nodes or white points in a certain area around a point. Thus nodes don't get on the verge of cracks, and are placed accurately on center that reflects layout of cracks more adequately. At this stage the user has an opportunity to set distance between points.

In case of nodal points connection, the most probable is calculated the direction of a crack. For this purpose, for each point search of the longest segment passing 
through it is run and is in crack section limits. The direction (angle) of a long segment is considered the most probable traveling direction of a crack for this nodal point. Further, on the basis of the analysis closeness of the received angles of crack distribution there are circuits of the interdependent points reflecting the direction and the provision of a crack.

Simultaneously with the segmentation the application sets up nodal points which allow to divide image into composing parts and to identify in such a way sections belonging to certain fractures. Nodal points are not set by certain step but in each pixel thus checking up for existing nodes or white dots in a certain area around the point. By connecting the nodal most likely direction of the fracture is figured. The resulting set of segments received by previous calculations may contain inconsistent parts or not fully reflect the position of fractures. So segments shorter than certain measure are removed. Selection of this measure of fracture's lengths is based on fractures density and unfiltered noise effects. At this point, the user is able to specify the segment length, which is not taken into account.

Thus, we can conclude that a median filter is effective in removing noise, as it leads to less distortion of analyzed objects borders. Christian's method of adaptive binarization most reliably detects and calculates the cutoff boundary cracks in the original image. Canny segmentation method provides comparatively best detection limits, but it requires much more time.

Stages of image processing by "Crack Stone" are resulted on Fig. 7.

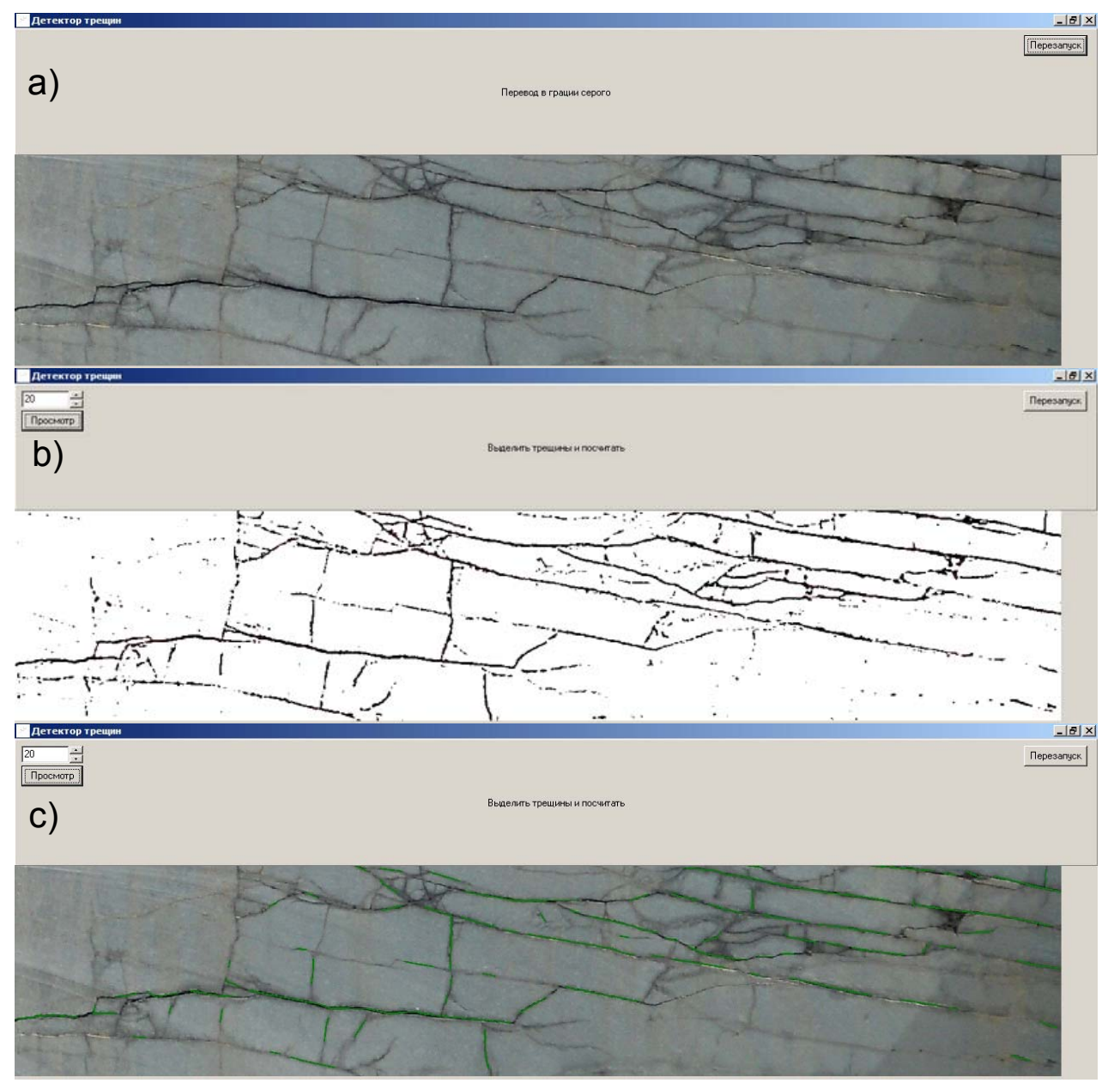

Fig. 7. Stages of image processing for cracks measurements by "Crack Stone": a) median filtration; b) adaptive binarization; c) segmentation. 
As a result of performance of all aforementioned stages of image processing, software developed by the author «Crack Stone» calculates the area of cracks, their quantity and length (Fig. 8). For the dimension stone rock mass of Bukinsky deposit, which are shown on Fig. 1, the cracks area is $0,023 \mathrm{~m}^{2}$, summary length of cracks with length more than $0,5 \mathrm{~m}-5,25 \mathrm{~m}$, quantity $-9 \mathrm{pc}$.

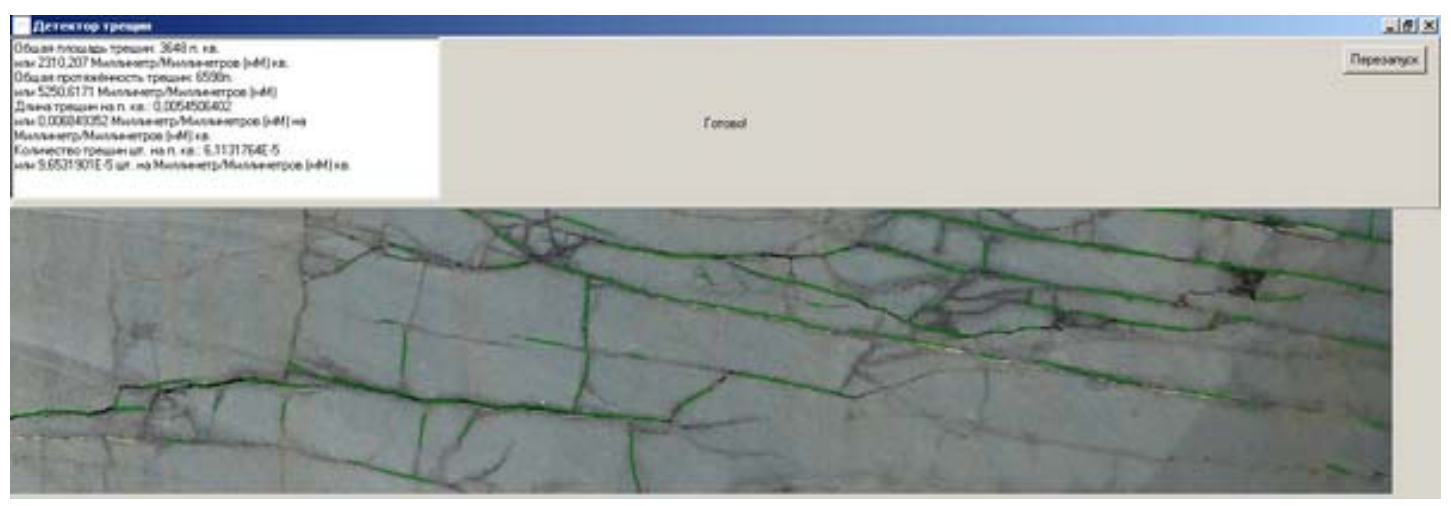

Fig. 8. Result of image processing of massif fracturing and calculating the area, quantity and length of cracks by "Crack Stone"

The algorithm of cracks search and analysis has a lot of input parameters, which directly influence on result of image processing by application. Therefore, it is important to estimate their influence on qualitative and quantity parameters of stone fracturing for the purpose of an exception of miscalculations, which provide the further researches in this direction.

\section{Conclusion}

Application of photogrammetric methods of fracturing parameters research on dimension stone quarries allows to identify cracks in rock masses with following calculation of their linear sizes and forecasting of out of block production on separate sites of a deposit. Creation of rock mass complex model which includes angular and linear sizes between cracks of adjacent systems, demands application of the modern high-precision equipment and the corresponding software. The further researches consist in a choice or creation of the rational software for the decision of the abovestated problems.

Remote rock mass mapping techniques are still a need to establish stringent mapping methodologies for efficient setup and operation of the various techniques. The results of the investigation show it is also necessary to establish guidelines for effective rock mass identification, to ensure that the necessary detail and scale effects are taken into consideration.

\section{Acknowledgements}

This work was supported by the Department of Photogrammetry, Remote Sensing and Geographic Information Systems of Warsaw University of Technology under the project Erasmus Mundus Action 2 Lot 5 ACTIVE. The author is very thankful prof. Dorota Zawieska and assist. prof. Jakub Markiewicz (GiK, Warsaw University of Technology) for the useful support. 


\section{References}

Canny, J. (1986). A computational approach to edge detection. IEEE Transactions on Pattern Analysis and Machine Intelligence, 8(6), pp. 679-698.

Coggan, J.S., Wetherelt, A., Gwynn, X.P. \& Flynn, Z.N. (2007). Comparison of handmapping with remote data capture systems for effective rock mass characterization. 11th Congress of the International Society for Rock Mechanics, 1, pp. 201-206.

Dare, P.M., Hanley, H.B., Fraser, C.S., Riedel, B. \& Niemeier, W. (2002). An operational application of automatic feature extraction the measurement of cracks in concrete structures. Photogrammetric Record, 17(99), pp. 453-464.

Delis P., Wojtkowska M., Nerc P., Ewiak I. \& Lada A. (2016). Integration of geodata in documenting castle ruins. XXIII ISPRS CONGRESS, COMMISSION III. International Archives of the Photogrammetry Remote Sensing and Spatial Information Sciences, 41(B3), pp. 345-349. doi:10.5194/isprsarchives-XLI-B3-3452016.

Feng, Q.H. \& Roshoff, K. (2004). In-Situ Mapping and Documentation of Rock Faces Using a Full Coverage 3D Laser Scanning Technique. International Journal of Rock Mechanics and Mining Science, 41(1), pp. 1-6.

Gonzalez, R.C. \& Woods, R.E. (2008). Digital image processing (3rd edition). Upper Saddle River, NJ: Prentice-Hall.

González-Aguilera, D., López-Fernández, L., Rodriguez-Gonzalvez, P., Guerrero, D., Hernandez-Lopez, D., Remondino, F., Menna, F., Nocerino, E., Toschi, I., Ballabeni, A. \& Gaiani, M. (2016). Development of an all-purpose free photogrammetric tool. The International Archives of the Photogrammetry, Remote Sensing and Spatial Information Sciences, 41(B6), pp. 31-38. DOI: 10.5194/isprsarchives-XLI-B6-31-2016.

Haneberg, W.C. (2008). Using close range terrestrial digital photogrammetry for 3-D rock slope modeling and discontinuity mapping in the United States. Bull. Eng. Geol. Environ, 67(4), pp. 457-469.

Jing, L. (2003). A review of techniques, advances and outstanding issues in numerical modelling for rock mechanics and rock engineering. International Journal of Rock Mechanics and Mining Sciences, 40(3), pp. 283-353.

Kemeny, J., Mofya, E., Holmlund, J. \& Ahlgren, S. (2002). Digital imaging for rock mass characterization. Proceedings of the 2nd Annual Conference on the Application of Geophysical and NDT Methodologies To Transportation Facilities and Infrastructure (Geophysics 2002), Los Angeles.

Kemeny, J. \& Post, R. (2003). Estimating three-dimensional rock discontinuity orientation from digital images of fracture traces. Computers and Geosciences, 29 (1), pp. 65-77. DOI: 10.1016/S0098-3004(02)00106-1.

Lemy, F. \& Hadjigeorgiou, J. (2003). Discontinuity trace map construction using photographs of rock exposures. International Journal of Rock Mechanics and Mining Sciences, 40(6), pp. 903-917.

Mohebbi, M., Yarahmadi Bafghi, A.R., Fatehi Marji M. \& Gholamnejad J. (2017). Rock mass structural data analysis using image processing techniques (Case study: Choghart iron ore mine northern slopes). Journal of Mining \& Environment, 8(1), pp. 61-74. DOI: 10.22044/jme.2016.629.

Poropat, G.V. (2001). New methods for mapping the structure of rock masses. CSIRO Exploration and Mining, paper for Explo 2001, pp. 253-260. 
Priest, S.D. (1993). Discontinuity analysis for rock engineering. Chapman \& Hall, London, 473.

Sobolevskyi, R., Zuievska, N., Korobiichuk, V., Tolkach O. \& Kotenko V. (2016). Cluster analysis of fracturing in the deposits of decorative stone for the optimization of the process of quality control of block raw material. EasternEuropean Journal of Enterprise Technologies, 5(83), pp. 21-29. DOI: $10.15587 / 1729-4061.2016 .80652$.

Turanboy, A. \& Ülker, E. (2010). A new approach to rapid 3D mapping of rock mass structure. Geotechnical Engineering, 163(6), pp. 321-331. DOI: 10.1680/geng.2010.163.6.321.

Wang, P. \& Huang, H. (2010). Comparison analysis on present image-based crack detection methods in concrete structures. 2010 3rd International Congress on Image and Signal Processing (CISP2010), 5, pp. 2530-2533.

Yamaguchi, T. \& Hashimoto, S. (2009). Practical image measurement of crack width for real concrete structure. Electronics and Communications in Japan, 92(10), pp. 605-614.

Zawieska, D. \& Markiewicz, J. (2015). Utilisation of laser scanning technology and digital images for measurements of industrial objects - a case study. Reports on Geodesy and Geoinformatics, 98(1). DOI: 10.2478/rgg-2015-0003.

\section{Author:}

Assist. Prof. Volodymyr Levytskyi, v.levytskyi@ztu.edu.ua

Department of Mine Surveying

Zhytomyr State Technological University

St. Chudnivska 103, 10005, Zhytomyr, Ukraine 